PROCEEDINGS OF THE

AMERICAN MATHEMATICAL SOCIETY

Volume 133, Number 12, Pages 3657-3661

S 0002-9939(05)07928-1

Article electronically published on June 6, 2005

\title{
CHARACTERISTIC NUMBERS OF POSITIVELY CURVED SPIN-MANIFOLDS WITH SYMMETRY
}

\author{
ANAND DESSAI \\ (Communicated by Jon G. Wolfson)
}

\begin{abstract}
Let $M$ be a Spin-manifold of positive sectional curvature and dimension $>8$. Suppose a compact connected Lie group $G$ acts smoothly on $M$. We show that the characteristic number $\hat{A}(M, T M)$ vanishes if $G$ contains two commuting involutions acting isometrically on $M$.
\end{abstract}

\section{INTRODUCTION}

Lichnerowicz showed that the Dirac operator of a closed Riemannian Spinmanifold $M$ of positive scalar curvature is invertible [12. In particular, if $\operatorname{dim} M=$ $4 m$, the characteristic number $1 \hat{A}(M)$ which is the index of the Dirac operator vanishes by the Atiyah-Singer index theorem [2]. Conversely, it is easy to see (cf. [10, p. 424) that any characteristic number which vanishes on all $4 m$-dimensional Spin-manifolds of positive scalar curvature is a multiple of the $\hat{A}$-genus.

In contrast, the question of which characteristic numbers vanish in the presence of positive sectional curvature is wide open. Of course the answer is trivial in dimension 4 since in this dimension any characteristic number is a multiple of the $\hat{A}$-genus. In dimension 8 the space of characteristic numbers is spanned by the $\hat{A}$-genus and the signature. Since the quaternionic plane (with its standard metric) has positive sectional curvature and non-vanishing signature, one finds again that any characteristic number obstructing positive sectional curvature is a multiple of the $\hat{A}$-genus. In dimension $4 m>8$, however, it is an open question as to which characteristic numbers obstruct positive sectional curvature on Spin-manifolds. In fact, besides the $\hat{A}$-genus, apparently the only other known restriction on the characteristic numbers is given by an upper bound on the absolute value of the signature, a consequence of Gromov's Betti number theorem 9 .

In this note we consider the characteristic number $\hat{A}(M, T M)$ which is the index of the Dirac operator twisted with the complexified tangent bundle (this operator is also known as the Rarita-Schwinger operator). Our main result asserts that $\hat{A}(M, T M)$ is an obstruction to positive sectional curvature under rather mild assumptions on the symmetry.

Received by the editors October 24, 2003 and, in revised form, July 8, 2004.

2000 Mathematics Subject Classification. Primary 53C20; Secondary 58J26.

Key words and phrases. Positive curvature, equivariant index theory, elliptic genera.

${ }^{1} \mathrm{~A}$ characteristic number is a linear combination of Pontrjagin numbers.

(C)2005 American Mathematical Society Reverts to public domain 28 years from publication 3657 
Theorem 1.1. Let $M$ be a closed connected Spin-manifold of dimension $>8$ and let $G$ be a compact connected Lie group which acts smoothly on $M$. Suppose $M$ admits a metric of positive sectional curvature such that some subgroup $\mathbb{Z} / 2 \times \mathbb{Z} / 2$ of $G$ acts effectively and isometrically. Then $\hat{A}(M)=\hat{A}(M, T M)=0$.

Note that the lower bound on the dimension is necessary since $\hat{A}(M, T M)$ does not vanish for $M$ the quaternionic projective plane. The author does not know whether the conclusion of the theorem holds without assumptions on the symmetry.

If the dimension of the isometry group Iso $(M)$ is greater than one, then the connected component of the identity contains a subgroup isomorphic to $\mathbb{Z} / 2 \times \mathbb{Z} / 2$. Hence, Theorem 1.1 implies

Corollary 1.2. Let $M$ be a closed connected Spin-manifold of dimension $>8$. If $M$ admits a metric of positive sectional curvature with $\operatorname{dim} \operatorname{Iso}(M)>1$, then $\hat{A}(M)=\hat{A}(M, T M)=0$.

In [6] it is shown that the conclusion also holds for 2-connected manifolds of positive sectional curvature with effective isometric $S^{1}$-action.

For a 12-dimensional manifold $M$ the theorem can be formulated in terms of the signature. In this dimension the signature satisfies

$$
\operatorname{sign}(M)=8 \hat{A}(M, T M)-32 \hat{A}(M) .
$$

This follows from a direct computation or by comparing the elliptic genus in different cusps.

Corollary 1.3. Let $M$ be a closed connected Spin-manifold of dimension 12 . If $M$ admits a metric of positive sectional curvature with $\operatorname{dim} \operatorname{Iso}(M)>1$, then $\hat{A}(M)=$ 0 and $\operatorname{sign}(M)=0$.

Example 1.4. Consider the Riemannian product of the quaternionic plane (with its standard metric) and a Ricci-flat $K_{3}$-surface. The manifold has positive scalar and non-negative Ricci curvature. Its signature is equal to \pm 16 . The author does not know whether this manifold admits a metric of positive sectional curvature. By the corollary above any such metric would be quite unsymmetrical.

\section{Proof of Theorem 1.1}

On the topological side the proof relies on the theory of elliptic genera. For later reference we shall briefly recall the relevant facts.

The elliptic genus $\Phi(M)$ of a Spin-manifold $M$ is a modular function which expands in one of its cusps as a series of indices of twisted Dirac operators

$$
\begin{gathered}
q^{-\operatorname{dim} M / 8} \cdot \hat{A}\left(M, \bigotimes_{n=2 m+1>0} \Lambda_{-q^{n}} T M \otimes \bigotimes_{n=2 m>0} S_{q^{n}} T M\right) \\
=q^{-\operatorname{dim} M / 8} \cdot(\hat{A}(M)-\hat{A}(M, T M) \cdot q+\ldots) .
\end{gathered}
$$

Here $\Lambda_{t}:=\sum \Lambda^{i} \cdot t^{i}$ (resp. $S_{t}:=\sum S^{i} \cdot t^{i}$ ) is the exterior (resp. symmetric) power operation and $\hat{A}(M, E)$ denotes the index of the Dirac operator twisted with the complexification of the vector bundle $E$. The expansion above determines $\Phi(M)$ and can be taken as a definition. 
The main feature of the elliptic genus is its rigidity under smooth $S^{1}$-actions conjectured by Witten and proved by Taubes and Bott-Taubes (see 4] and references therein). The rigidity theorem allows us to study finite cylic subactions of $S^{1}$ in terms of the expansion above [11, 7]. For further reference we point out the following consequences for involutions due to Hirzebruch and Slodowy.

Proposition 2.1 (11]). Suppose $M$ is a connected Spin-manifold with smooth $S^{1}$ action and the element $\sigma \in S^{1}$ of order 2 acts effectively. If $\hat{A}(M, T M) \neq 0$, then the action is even and the fixed point manifold $M^{\sigma}$ has codimension 4.

Here the codimension of $M^{\sigma}$ is defined to be the minimal codimension of its connected components, and the $S^{1}$-action is even if the codimension of each component of $M^{\sigma}$ is divisible by 4 (otherwise the action is odd). For an odd action the elliptic genus vanishes identically. This is a direct consequence of the rigidity.

In [1] Hirzebruch and Slodowy used the rigidity theorem and the Lefschetz fixed point formula [3] to compute $\Phi(M)$ in terms of the elliptic genus of the transversal self-intersection $M^{\sigma} \circ M^{\sigma}$ of the fixed point manifold $M^{\sigma}$, i.e. $\Phi(M)=\Phi\left(M^{\sigma} \circ M^{\sigma}\right)$. The second part of the proposition follows from this identity and expansion (11).

On the geometric side the proof of Theorem 1.1 is based on the following two properties of totally geodesic submanifolds. The first is an old result of Frankel.

Intersection Theorem 2.2 ([8]). Let $M$ be a connected Riemannian manifold of positive sectional curvature. Suppose $N_{1}$ and $N_{2}$ are totally geodesic submanifolds. If $\operatorname{dim} N_{1}+\operatorname{dim} N_{2} \geq \operatorname{dim} M$, then $N_{1}$ and $N_{2}$ intersect, i.e. $N_{1} \cap N_{2} \neq \emptyset$.

To prove this statement one uses a Synge-type argument for a geodesic minimizing the distance between $N_{1}$ and $N_{2}$. Recently, Wilking has shown among other things that the inclusion map of totally geodesic submanifolds in positive curvature is highly connected.

Connectivity Theorem 2.3 ([13]). Let $M$ be a connected Riemannian manifold of positive sectional curvature. Suppose $N$ is a connected totally geodesic submanifold of codimension $k$. Then the inclusion $j: N \hookrightarrow M$ is (dim $M-2 k+1)$-connected.

This statement follows from a Morse-type argument for the space of path in $M$ starting and ending in $N$.

Let $u:=j_{!}(1) \in H^{k}(M ; \mathbb{Z})$, where $j_{!}: H^{*}(N ; \mathbb{Z}) \rightarrow H^{*+k}(M ; \mathbb{Z})$ denotes the pushforward in cohomology (defined using Poincaré duality). Then by the Connectivity Theorem

$$
\cup u: H^{*}(M ; \mathbb{Z}) \rightarrow H^{*+k}(M ; \mathbb{Z})
$$

is injective for $k-1<* \leq n-2 k+1$ and surjective for $k-1 \leq *<n-2 k+1$ [13. The same holds true if we replace $\mathbb{Z}$-coefficients by $\mathbb{Z} / 2$-coefficients.

Note that for any isometry $\sigma$ of $M$ the fixed point manifold $M^{\sigma}$ is totally geodesic. In the proof of Theorem 1.1 we shall apply the results above to the connected components of $M^{\sigma}$, where $\sigma$ is an involution.

Proof of Theorem 1.1. The proof is by contradiction. So assume $\hat{A}(M, T M) \neq 0$. In particular, $\operatorname{dim} M=4 m \geq 12$. Note that $M$, being an even-dimensional oriented manifold of positive sectional curvature, is simply connected by the classical Synge theorem.

Let $H \cong \mathbb{Z} / 2 \times \mathbb{Z} / 2$ be a subgroup of $G$ which acts effectively and isometrically on $M$. Since $G$ is connected, every element of $H$ is contained in some $S^{1}$-subgroup 
of $G$. Let $\sigma_{1}, \sigma_{2}, \sigma_{3}$ denote the non-trivial involutions in $H$. By Proposition 2.1 the $\sigma_{i}$-action is even and the fixed point manifold $M^{\sigma_{i}}$ has codimension 4 . Using Intersection Theorem 2.2 we see that $M^{\sigma_{i}}$ is the union of a codimension-4 component $F_{i}$ and a possibly empty set of isolated $\sigma_{i}$-fixed points. By Connectivity Theorem $2.3 F_{i}$ is simply connected.

Since $H$ acts effectively any fixed point $p t \in M^{H}$ must belong to two different codimension- 4 components, i.e. $p t \in F_{s} \cap F_{t}$ for some $s \neq t$. Since $\operatorname{dim} M \geq 12$ it follows that $M^{H}$ does not contain any isolated $\sigma_{i}$-fixed points and $M^{H}=\bigcap_{i} F_{i}$.

The action of $H$ on $T_{p t} M$ splits into one-dimensional real $H$-representations. Recall that for any non-trivial involution of $H$ the fixed point component which contains $p t$ has codimension 4. From this it is straightforward to check that the subspace $\left(T_{p t} M\right)^{H}$ of $T_{p t} M$ has codimension 6, i.e. the connected component of $M^{H}$ containing $p t$ has codimension 6 in $M$. Since $\operatorname{dim} M \geq 12$, we see from Intersection Theorem 2.2 that $M^{H}$ is connected.

We now apply the cohomological implication of Connectivity Theorem 2.3 to the totally geodesic submanifold $j: M^{H} \hookrightarrow F_{1}$ (which has codimension 2) to conclude that multiplication with $u=j_{!}(1) \in H^{2}\left(F_{1} ; \mathbb{Z} / 2\right)$ gives an isomorphism $H^{2 i}\left(F_{1} ; \mathbb{Z} / 2\right) \rightarrow H^{2 i+2}\left(F_{1} ; \mathbb{Z} / 2\right)$ for $0<2 i \leq 4 m-8$ and that $H^{2 *+1}\left(F_{1} ; \mathbb{Z} / 2\right)=0$. In particular, either $u=0$ and $F_{1}$ is a $\mathbb{Z} / 2$-cohomology sphere or $u \neq 0$ and $H^{*>0}\left(F_{1} ; \mathbb{Z} / 2\right)$ is generated by $H^{2}\left(F_{1} ; \mathbb{Z} / 2\right)$.

We claim that the second case does not occur. To see this we inspect the LeraySerre spectral sequence for the Borel construction

$$
F_{1} \stackrel{k}{\hookrightarrow}\left(F_{1}\right)_{\mathbb{Z} / 2} \rightarrow B \mathbb{Z} / 2,
$$

where $\mathbb{Z} / 2$ denotes the group generated by $\sigma_{2}$. Since $F_{1}$ is simply connected and $\left(F_{1}\right)^{\mathbb{Z} / 2}=M^{H}$ is not empty $k^{*}: H^{*}\left(\left(F_{1}\right)_{\mathbb{Z} / 2} ; \mathbb{Z} / 2\right) \rightarrow H^{*}\left(F_{1} ; \mathbb{Z} / 2\right)$ is surjective in degree $* \leq 2$. Assuming $u \neq 0$ it follows that $k^{*}$ is surjective in all degrees which in turn implies (see [5], Ch. VII)

$$
H^{*}\left(F_{1} ; \mathbb{Z} / 2\right) \cong H^{*}\left(M^{H} ; \mathbb{Z} / 2\right) .
$$

On the other hand, since $4 m \geq 12$ and $M^{H} \hookrightarrow F_{1}$ is $(4 m-7)$-connected, we have $H^{2 *+1}\left(M^{H} ; \mathbb{Z} / 2\right)=0, H^{2}\left(F_{1} ; \mathbb{Z} / 2\right) \cong H^{2}\left(M^{H} ; \mathbb{Z} / 2\right)$ and multiplication with $u_{\mid M^{H}} \in H^{2}\left(M^{H} ; \mathbb{Z} / 2\right)$ gives an isomorphism $H^{2 i}\left(M^{H} ; \mathbb{Z} / 2\right) \rightarrow H^{2 i+2}\left(M^{H} ; \mathbb{Z} / 2\right)$ for $0<2 i \leq 4 m-10$. Hence,

$$
\operatorname{dim} H^{*}\left(F_{1} ; \mathbb{Z} / 2\right)=\operatorname{dim} H^{*}\left(M^{H} ; \mathbb{Z} / 2\right)+\operatorname{dim} H^{2}\left(F_{1} ; \mathbb{Z} / 2\right) .
$$

Comparing the last two display formulas we see that $H^{2}\left(F_{1} ; \mathbb{Z} / 2\right)=0$ and that $F_{1}$ as well as $M^{H}$ are $\mathbb{Z} / 2$-cohomology spheres (for a different argument which also gives information for submanifolds of higher codimension, see [13, Theorem 5.1). Using Connectivity Theorem 2.3 applied to $F_{1} \hookrightarrow M$ we see that $M$ is a $\mathbb{Z} / 2$-cohomology sphere as well. In particular, $\hat{A}(M, T M)=0$. This completes the proof of the theorem.

\section{ACKNOWLEDGEMENT}

I thank the referee for carefully reading the paper and for pointing out an incorrect argument in an earlier version. 


\section{REFERENCES}

[1] M.F. Atiyah and F. Hirzebruch, Spin-Manifolds and Group Actions, in: Essays on Topology and Related Topics. Georges de Rham, Springer (1970), 18-28 MR0278334 (43:4064)

[2] M.F. Atiyah and I.M. Singer, The index of elliptic operators. I, Ann. of Math. 87 (1968), 484-530 MR0236950(38:5243)

[3] M.F. Atiyah and I.M. Singer, The index of elliptic operators. III, Ann. of Math. 87 (1968), 546-604 MR0236952 (38:5245)

[4] R. Bott and C.H. Taubes, On the rigidity theorems of Witten, J. of Amer. Math. Soc. 2 (1989), 137-186 MR0954493 (89k:58270)

[5] G. Bredon, Introduction to compact transformation groups, Pure and Applied Mathematics, Vol. 46. Academic Press (1972) MR0413144 (54:1265)

[6] A. Dessai, Obstructions to positive curvature and symmetry, preprint, available at the arXiv: http://arxiv.org/abs/math.DG/0104256

[7] A. Dessai, Cyclic actions and elliptic genera, preprint, available at the arXiv: http://arxiv.org/abs/math.GT/0104255

[8] T. Frankel, Manifolds with positive curvature, Pacific J. Math. 11 (1961), 165-174 MR0123272 (23:A600)

[9] M. Gromov, Curvature, diameter and Betti numbers, Comment. Math. Helvetici 56 (1981), 179-195 MR0630949 (82k:53062)

[10] M. Gromov and H.B. Lawson, The classification of simply-connected manifolds of positive scalar curvature, Ann. of Math. 111 (1980), 209-230 MR0577131 (81h:53036)

[11] F. Hirzebruch and P. Slodowy, Elliptic Genera, Involutions and Homogeneous Spin Manifolds, Geom. Dedicata 35 (1990), 309-343 MR1066570 (92a:57028)

[12] A. Lichnerowicz, Spineurs harmoniques, C. R. Acad. Sci. Paris 257 (1963), 7-9 MR0156292 $(27: 6218)$

[13] B. Wilking, Torus actions on manifolds of positive sectional curvature, Acta Math. 191 (2003), 259-297 MR:2051400

Department of Mathematics, University of Münster, D-48149 Münster, Germany

E-mail address: dessai@math.uni-muenster.de

$U R L:$ http://wwwmath.uni-muenster.de/math/u/dessai 